\title{
Changes in gait when anticipating slippery floors
}

\author{
Rakié Cham $^{\mathrm{a}, *}$, Mark S. Redfern ${ }^{\mathrm{a}, \mathrm{b}}$ \\ ${ }^{a}$ Room 153, Department of Bioengineering, Eye and Ear Institute Building, University of Pittsburgh, 203 Lothrop street, Pittsburgh, \\ PA 15213, USA \\ ${ }^{\mathrm{b}}$ Department of Otolaryngology, University of Pittsburgh, Pittsburgh, PA, USA
}

Accepted 8 June 2001

\begin{abstract}
Falls precipitated by slipping are listed among the leading causes of injuries. The biomechanical analysis of such events is a necessary component of the slips/falls prevention research. One of the challenges of biomechanical studies is reproducing the unexpected nature of real-life slipping accidents. Thus, the goal of this study was to quantify changes in gait biomechanics when subjects anticipate slippery environments. Foot ground reaction forces and body dynamics of 16 subjects were recorded during level walking and descending ramps of varying frictional properties and inclination. Gait biomechanics were compared among three types of dry trials: (1) baseline (subjects knew the floor was dry); (2) anticipation (subjects were uncertain of the contaminant condition, dry, water, soap or oil); and (3) recovery trials recorded after a contaminated trial (subjects again knew the floor was dry). Subjects were asked to walk as naturally as possible throughout testing. Anticipation trials produced peak required coefficient of friction $\left(\mathrm{RCOF}_{\text {peak }}\right)$ values that were on average 16-33\% significantly lower than those collected during baseline trials, thus reducing slip potential. During recovery trials, $\mathrm{RCOF}_{\text {peak }}$ values did not return to baseline characteristics $(5-12 \%$ lower). Postural and temporal gait adaptations, which affected ground reaction forces, were used to achieve $\mathrm{RCOF}_{\text {peak }}$ reductions. Statistically significant gait adaptations included reductions in stance duration (SD) and loading speed on the supporting foot, shorter normalized stride length (NSL), reduced foot-ramp angle and slower angular foot velocity at heel contact. As a result of these adaptations, anticipation of slippery surfaces led to significant changes in lower extremity joint moments, a reflection of overall muscle reactions. Thus, this study suggests that significant gait changes are made when there is a potential risk of slipping even though subjects were asked to walk as naturally as possible. Insights are also gained into the adaptations that are used to reduce the potential of slips/falls. (C) 2002 Elsevier Science B.V. All rights reserved.
\end{abstract}

Keywords: Gait adaptations; Anticipation of slippery floors; Slips and falls biomechanics

\section{Introduction}

Falls are a major cause of serious injuries and even deaths. More than $20 \%$ of injury-related emergency department visits have been attributed to falls, the single largest cause of such visits [1]. The US economic cost of falling accidents ranks second in the USA [2]. The cost of falling injuries is also substantial among occupational populations for a number of reasons including the advancing age of the labor force [3] but also the severity of the injuries, with more than $25 \%$ of the workers sustaining falling injuries missing 31 days or

\footnotetext{
* Corresponding author. Tel.: + 1-412-647-8050.
}

E-mail address: chamr@msx.upmc.edu (R. Cham). more at work [4]. In a comparative study on analysis of injury mortality data across industrial countries, Fingerhut et al. frequently listed falling among the three leading generators of fatalities [5]. The loss of balance leading to falls is often the result of slipping events [6]. The US National Health Interview Survey questionnaire of 1997 revealed a clear majority $(64 \%)$ of the work-related falls attributed to slipping, tripping or stumbling. The 1992-1998 occupational same level fatal falls records providing narrative description of the incident indicated that slipping was the most common triggering event ( $43 \%$ of the cases). In 1998, slips, trips and falls accounted for $16.8 \%$ of all nonfatal occupational injuries involving days away from work and $11.9 \%$ of job-related deaths [4]. The incidence of slip accidents varies with geographical location and external environmental conditions. For example, it is particu- 
larly high among occupational populations (e.g. miners and mail deliverers) working outside during cold winter months $[7,8]$. In Sweden, Björnstig et al. found the cost of medical care inflicted by slipping accidents on ice and snow comparable with the cost of all traffic injuries in that same area [9].

Findings of biomechanical gait experiments have been used in an effort to understand human factors that cause slips/falls accidents and their complex interaction with environmental factors [10-14]. Gait biomechanics and the health of the sensory and neuromuscular systems are included in human factors. Among the most important environmental factors are the frictional and material properties of the shoes and floors. One important example of the interaction between human and environmental factors is the increased risk of slipping as the frictional requirements of the task performed (walking, load carrying, pushing/pulling) exceed the available frictional capabilities of the shoe/floor interface, i.e. the measured coefficient of friction [12]. Thus, the frictional requirements of a particular task measured in slips/falls experiments are useful in setting thresholds of minimal friction needed to avoid a slip and determining whether an environment is 'slip-safe'. In addition, relevant gait variables generated from biomechanical slips/falls studies have been employed in the development of a new generation of slip resistance testers that measure the frictional properties of the shoe/floor interface by simulating foot movements during locomotion [15-17].

The use of biomechanical gait studies in slips/falls prevention research goes beyond measuring slip resistance and setting frictional thresholds to achieve slipsafe environments. Gait biomechanics partially determine the outcome of walking onto a contaminated floor and are reflection of the ability of the human neuromotor system to: (1) decrease slip potential in possibly dangerous environments (before a slip occurs); and (2) recover from a slip event by generating corrective reactions in an attempt to avoid a fall. The success of the human neuromotor system in achieving those goals will be affected by various factors such as anthropometry, strength, age, task (physical constraints) and cognitive/behavioral factors (mental stress, reaction time, attention, fear of falling). Thus, biomechanical studies are a valuable clinical tool to investigate the impact of each of those factors on the ability to decrease slip potential and recover balance after a slip event.

A challenge in slip/fall biomechanical studies has been to reproduce the unexpected nature of real-life slipping accidents in laboratory settings. The effect of anticipating potentially slippery surfaces on gait biomechanics has not been investigated. Andres et al. [18] have compared the kinematic characteristics of steps prior to and onto a targeted known slippery area. However, the subjects knew the surface was slippery.
Examining possible gait adaptations arising from anticipation effects will have implications on the findings of slip/fall experiments and provide insights on 'control mechanisms' used to reduce slip and fall potentials.

The goal of this study was to investigate whether subjects change gait biomechanics (on both level and inclined surfaces) when there is a possibility of a slippery environment. Specific changes in walking patterns (if any) adopted were quantified. The findings reported here (dry surfaces) are part of a larger study that investigated gait biomechanics on different surfaces of varying inclination and slipperiness (dry, wet, soap, oil).

\section{Methods}

\subsection{Subjects}

Sixteen healthy subjects (eight male, eight female) participated in this study with informed consent approved by the Institutional Review Board of the University of Pittsburgh. Their ages ranged from 19 to 30 years (mean 23 years, S.D. 4 years), mean weight from 62.6 to $82.4 \mathrm{~kg}$ (mean $68.7 \mathrm{~kg}$, S.D. $6.8 \mathrm{~kg}$ ) and height from 1.63 to $1.85 \mathrm{~m}$ (mean $1.73 \mathrm{~m}$, S.D. $0.07 \mathrm{~m}$ ). Exclusionary criteria included a history of neurological or orthopedic disease and any difficulties impeding normal locomotion.

\subsection{Experimental set-up}

A specially designed walkway was used to conduct these experiments [11]. It consists of a ramp attached on one end to an electro-mechanical platform that can be raised or lowered to easily change ramp angle, and at the opposite end, this ramp is hinged to an extension of the walkway. This extension is the same height as the lowest level of the platform to attain a $0^{\circ}$-walkway. The ramp is $1.8-\mathrm{m}$ long and $1.0-\mathrm{m}$ wide with a $1.4-\mathrm{m}$ extension at the bottom. A force platform (Bertec, Inc.) is built into the ramp to record ground reaction forces. This force plate is bolted to the superstructure of the ramp and positioned such that the subjects' left foot landed on the platform during the second or third step of descending the ramp. The top surface of the ramp is made of $1.9-\mathrm{cm}$ thick plywood that is bolted to the frame, but is easily removed to allow the relatively fast changing of flooring conditions.

The data acquisition system consisted of the force plate, analog to digital (A/D) converter, PC computer and an OPTOTRAK 3020-motion measurement system. A graphical programming software (LABVIEW) was used to synchronize and collect force plate and motion data at $350 \mathrm{~Hz}$. The OPTOTRAK system recorded 3-D whole body and foot motion by tracking LEDs attached to the subject with an accuracy better 
than $1 \mathrm{~mm}$. More specifically, the LEDs were attached to the left shoulder (acromion), hip (greater trochanter), knee (lateral femoral condyle), ankle (lateral malleolus) and shoe (three markers near the heel of the shoe and the fifth metatarsal level on the lateral side).

\subsection{Experimental conditions and design}

\subsubsection{Conditions}

The primary independent variables included ramp angle, floor type and trial type. The three ramp angles used in this experiment were 0,5 and $10^{\circ}$. Subjects walked on (1) vinyl tile; (2) smooth painted plywood and (3) a rough, silicate impregnated, painted plywood floors. The findings of three types of trials are reported here, baseline, anticipation (dry) and recovery. In the baseline and recovery trials, subjects were certain that the floor was dry and, therefore, not slippery. The difference between these two types was that recovery trials occurred after a contaminated condition (wet, soap or oil), whereas baseline trials were collected at the beginning of a testing session before any slip occurred. In the anticipation trials, the subjects did not have a priori knowledge of the contaminant condition, but were told that there could be a contaminant. All subjects wore the same model of shoes with polyvinyl chloride (PVC) hard-soles for all trials.

\subsubsection{Experimental design}

A full factorial design was used such that each subject was tested on all 27 conditions ( 3 ramp angles $\times 3$ floors $\times 3$ trial types). Subjects returned for a total of three visits, each time to be tested on a randomly chosen floor. The order of the conditions for each testing day was as follows: first, five baseline trials on each ramp angle were collected; then the ramp was set to the first angle at which the subject was to be tested; each of the three contaminated conditions (randomized) was 'hidden' among one to three 'anticipation dry' trials and followed by three 'recovery' trials. This protocol was then repeated for the other two ramp angles (the order of presentation of the ramp angles was randomized).

\subsubsection{Walking protocol}

First, an overview of the experiment (general goals, walking protocol, trial types, contaminants and variables recorded) was verbally described to the subject and informed consent obtained. LEDs were placed on the left side of the body and foot. After being equipped with the safety harness to prevent injury from falling should a slip occur, the subject was instructed to walk at a comfortable pace throughout the experiment and as naturally as possible even though there may be a slippery condition (the importance of these specific instructions was emphasized). Subjects were also reassured that they would be caught in a case of a slip. Each subject was first allowed to become familiar with the ramp set-up by walking across the force plate such that the left foot hits the force plate area. For the baseline trials, the subject walked to the top of the ramp, turned around and walked down the ramp. Prior to each anticipation trial, the subject walked to the starting line of the walkway, faced away from the walkway, waited for about $1 \mathrm{~min}$ while listening to loud music, distracting him/her from any possible contaminant application on the floor. During this waiting period, a contaminant was applied to the surface of the force plate if required for that trial (the lighting in the room was dimmed throughout the experiment so that subjects could not perceive the applied contaminant (if any)). At the end of the waiting period, the lights in the room were turned on and off, a signal for the subject to stop the music, turn around and walk down the ramp, while looking straight ahead at the wall on the opposite side of the room. Upon completing the trial, if the floor surface was contaminated, the subject was seated and the shoes/floor were either changed or cleaned. Mistrials with subjects not fully contacting the force plate occurred in only $10 \%$ of the trials.

\subsection{Data processing and analysis}

Time was normalized to stance duration (SD), with $0 \%$ being heel contact (HC) and 100\% representing toe-off the force plate. Ground reaction forces and body/foot motion data were used to calculate specific gait variables used as the dependent variables (Table 1). Shear (anterior-posterior along the ramp direction) and normal (perpendicular to the ramp direction) ground reaction forces were normalized to the subject's weight. The ratio of shear to normal ground reaction, (termed the required coefficient of friction (RCOF) [11]) was computed for each trial. In addition, ankle, knee and hip moments were derived. Kinematic variables in the sagittal plane included foot-ramp angle obtained from the heel and toe position data, and shank-ramp angle computed from the knee and ankle position data. These variables were evaluated at $\mathrm{HC}$. Velocity information was calculated by numerically differentiating (using a two-time step differentiation routine) the position data provided by each LED. Velocity variables were the linear velocity of the heel along the ramp direction and the angular velocity of the foot as it rotated down onto the ramp floor. Both the linear heel velocity and angular foot velocity were evaluated at HC. In addition, maximum linear heel velocity in both rearward and forward directions recorded shortly after $\mathrm{HC}$ were also considered. Position data were filtered (least square low pass filter with cutoff frequency of 12 $\mathrm{Hz}$ ) only to derive acceleration variables used in lower 
extremity joint moments calculations. Finally, general gait variables such as normalized (to shank length) stride length (defined as the distance traveled in two steps from $\mathrm{HC}$ to $\mathrm{HC}$ ) and SD were considered in the analysis.

Due to the significantly different gait biomechanics on surfaces of varying inclination [11], the statistical analysis was conducted within each ramp angle condition. A within-subject repeated measures ANOVA of the effect of the independent variables (trial type, floor, trial type $\times$ floor) on each of the dependent variables (Table 1) was performed separately for each dependent variable. When the dependent variable was significantly affected by the trial type or floor type conditions, pairwise Tukey comparison tests were used to further investigate the differences in this dependent variable among the various trial and floor conditions. A significance level of $P \leq 0.01$ was used throughout the analysis except when otherwise specified.

Table 1

Definitions/abbreviations of dependent estimated parameters

\begin{tabular}{|c|c|}
\hline \multicolumn{2}{|c|}{ Kinetic parameters } \\
\hline Norm $_{\text {peak }}$ & $\begin{array}{l}\text { Peak of the first phase of the normal ground } \\
\text { reaction force curve }\end{array}$ \\
\hline Shear $_{\text {peak }}$ & $\begin{array}{l}\text { Peak of the first phase of the anterior-posterior } \\
\text { shear ground reaction force curve }\end{array}$ \\
\hline $\mathrm{RCOF}_{\text {peak }}$ & $\begin{array}{l}\text { Peak of the first phase of the required } \\
\text { coefficient of friction (RCOF) curve }\end{array}$ \\
\hline Norm $_{\text {Tpeak }}$ & Timing of Norm $_{\text {peak }}$ in percent of stance \\
\hline Shear $_{\text {Tpeak }}$ & Timing of Shear ${ }_{\text {peak }}$ in percent of stance \\
\hline Forc $_{\text {Tdiff }}$ & $\begin{array}{l}\text { Time difference between peaks of normal and } \\
\text { shear ground reaction forces } \\
\left(\text { Norm }_{\text {Tpeak }}-S_{\text {Shear }} \text { Tpeak }\right.\end{array}$ \\
\hline $\mathrm{RCOF}_{\text {Tpeak }}$ & Timing of $\mathrm{RCOF}_{\text {peak }}$ in percent of stance \\
\hline AnklMom $_{\text {peak }}$ & $\begin{array}{l}\text { Peak ankle dorsiflexion moment, i.e. peak of } \\
\text { the first phase of the ankle moment curve }\end{array}$ \\
\hline KneeMom $_{\text {peak }}$ & $\begin{array}{l}\text { Peak knee extension moment, i.e. peak of the } \\
\text { first phase of the knee moment curve }\end{array}$ \\
\hline HipMom $_{30}$ & $\begin{array}{l}\text { Hip flexion moment evaluated at 30\% into } \\
\text { stance }\end{array}$ \\
\hline \multicolumn{2}{|c|}{ Kinematic parameters } \\
\hline NSL & Normalized (to shank length) stride length \\
\hline SD & Stance duration \\
\hline HeelVelx $_{\mathrm{HC}}$ & Heel velocity in the direction of motion at $\mathrm{HC}$ \\
\hline HeelVelx $_{\text {rearward }}$ & $\begin{array}{l}\text { Peak rearward heel velocity in the direction of } \\
\text { motion, recorded shortly after HC }\end{array}$ \\
\hline HeelVelx $_{\text {forward }}$ & $\begin{array}{l}\text { Peak forward heel velocity in the direction of } \\
\text { motion, recorded shortly after HC }\end{array}$ \\
\hline HeelAccx $_{\mathrm{HC}}$ & $\begin{array}{l}\text { Heel acceleration in the direction of motion at } \\
\text { HC }\end{array}$ \\
\hline HeelAccx $_{\text {peak }}$ & $\begin{array}{l}\text { Peak heel acceleration in the direction of } \\
\text { motion, recorded shortly after HC }\end{array}$ \\
\hline FootAngVel $_{\mathrm{HC}}$ & Foot angular velocity at $\mathrm{HC}$ \\
\hline FootAngl $_{\mathrm{HC}}$ & Foot-ramp angle at $\mathrm{HC}$ \\
\hline ShankAngl $_{\mathrm{HC}}$ & Shank-ramp angle at $\mathrm{HC}$ \\
\hline
\end{tabular}
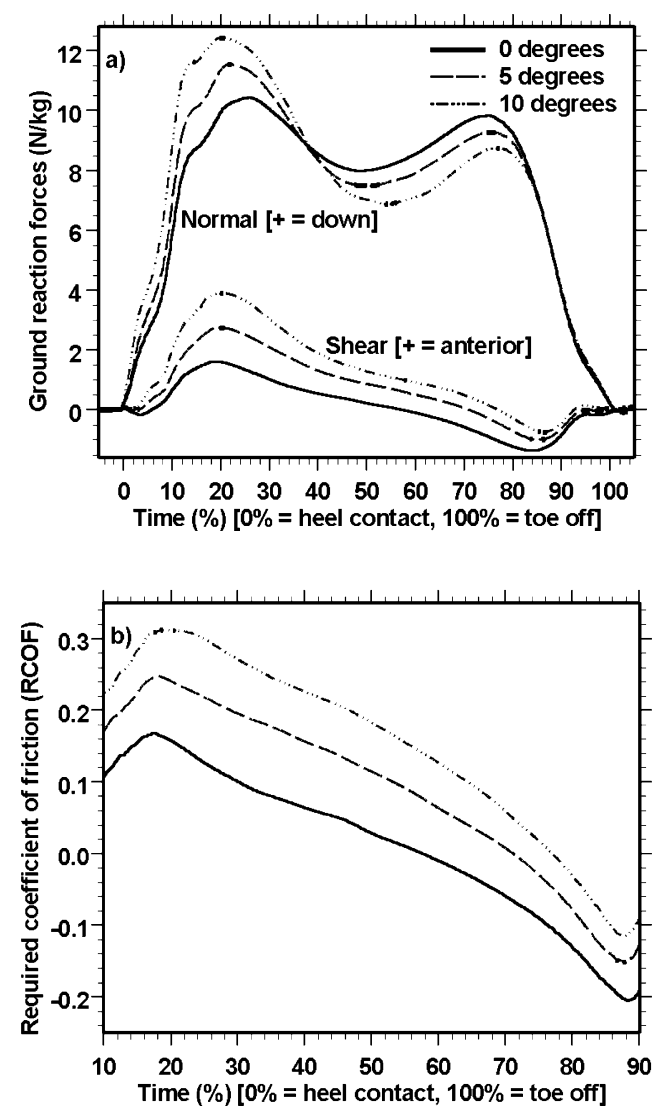

Fig. 1. Characteristic profile of ground reaction forces during gait on the vinyl tile floor $\left(0^{\circ}, 5^{\circ}, 10^{\circ}\right)$, averaged across all vinyl baseline trials, (a) Normal and anterior-posterior shear force (normalized to body weight); (b) required coefficient of friction, i.e. RCOF (shear/ normal ratio) (in Fig. 1b, time is truncated to $10-90 \%$ to avoid 'instability' region when both shear and normal forces are near 0 ).

\section{Results}

\subsection{Baseline data and ramp angle effect}

In order to investigate the anticipation effect of slippery surfaces on gait biomechanics, baseline normative values on horizontal and inclined vinyl surfaces were first recorded. As anticipated, the kinetics of locomotion in these baseline trials were affected by ramp angle [11]. More specifically, increases in the ramp angle were associated with increases in ground reaction forces and RCOFs (Fig. 1). For level walking, the typical biphasic shear force reached a maximum of 1.8 $\mathrm{N} / \mathrm{kg}$ and increased by about $60-70$ and $125-135 \%$ on 5 and $10^{\circ}$, respectively, $\left(\right.$ Shear $_{\text {peak }}$ in Table 2). The peak shear forces occurred at about $19 \%$ of stance for all ramp angles (Shear ${ }_{\text {Tpeak }}$ in Table 2). Peak normal forces increased by nearly $1 \mathrm{~N} / \mathrm{kg}\left(\right.$ Norm $_{\text {peak }}$ in Table 2$)$ and occurred earlier into stance as ramp angle was increased $\left(\right.$ Norm $_{\text {Tpeak }}$ in Table 2). The earlier occurrence of the peak normal force on inclined surfaces was also evident in the phase relationship of the ground reaction 
forces ( Forc $_{\text {Tdiff }}$ in Table 2). As a result of changes in these force profiles, the peak $\mathrm{RCOF}\left(\mathrm{RCOF}_{\text {peak }}\right.$ in Table 2), occurred about the same time $\left(\mathrm{RCOF}_{\text {Tpeak }}\right.$ in Table 2) as the peak shear force and increased from about 0.2 to 0.3 when changing the ramp angle from 0 to $10^{\circ}$.

Recorded kinematics showed that at the end of the swing phase, the heel rapidly decelerated (Fig. 2a-b and HeelAccx ${ }_{\mathrm{HC}}$ in Table 2) and, upon HC, a slight sliding motion of the heel occurred along the ramp surface. Different sliding patterns at or shortly after $\mathrm{HC}$ were observed. In general, the $\mathrm{HC}$ heel velocity was positive as noted in Fig. $2 \mathrm{a}$ and Table $2\left(\right.$ HeelVel $_{\mathrm{HC}}$ ), indicating a forward motion of the foot as it hit the floor. Then the heel slowed down rapidly and often even reversed direction with a mean peak rearward heel velocity of about $-0.1 \mathrm{~m} / \mathrm{s}$ (HeelVelx $\mathrm{x}_{\text {rearward }}$ reached at about $1-2 \%$ into stance or $6-12 \mathrm{~ms}$ after $\mathrm{HC}$ ). After this rearward motion, the heel slid forward again with an average peak forward heel velocity of about 0.12 $0.17 \mathrm{~m} / \mathrm{s}$ (HeelVelx forward $_{\text {was }}$ recorded at about $4-5 \%$ into stance or $24-33 \mathrm{~ms}$ after $\mathrm{HC}$ and was greater on inclined surfaces) and finally came to a stop (at about $6-7 \%$ into stance or $40-50 \mathrm{~ms}$ after $\mathrm{HC}$ ). However, there were also a significant number of trials where the velocity at $\mathrm{HC}$ was negative, i.e. foot moving in the rearward direction. In all cases, this rapid heel motion ended shortly after $\mathrm{HC}$ and the heel came to a complete stop. During this short heel motion time period after $\mathrm{HC}$, average peak heel acceleration $\left(\mathrm{HeelAcc}_{\text {peak }}\right.$ in Table 2) values of about $3-4 \mathrm{~m} / \mathrm{s}^{2}$ were recorded. The foot rotated down on the floor rapidly during this period (FootAngVel ${ }_{\mathrm{HC}}$ in Table 2) to reach foot-flat position at about $15 \%$ into stance (Fig. 2c) and the shank proceeded in its forward rotation (Fig. 2d).

\subsection{Anticipation effect on gait variables}

In general, ground reaction forces and motion data profiles for the anticipation trials were similar in shape to those in the baseline trials. However, ANOVA revealed trial type significantly $(P \leq 0.01)$ affected the magnitude and timing of gait, more specifically the ground reaction forces variables $\left(\mathrm{RCOF}_{\text {peak }}\right.$, $\mathrm{RCOF}_{\text {Tpeak }}$, Norm $\mathrm{Neak}_{\text {peak }}$ Norm $\mathrm{N}_{\text {Tpeak }}$, Shear $r_{\text {peak }}$, Shear Tpeak $_{\text {, }}$, and lower extremity kinematics (HeelVelx $_{\text {rearward }}$, HeelVelx forward $_{\text {, HelAccx }}$ peak, FootAngVel $_{\mathrm{HC}}$, FootAngl $_{\mathrm{HC}}$ and ShankAngl $\mathrm{HC}_{\mathrm{C}}$ ). Other variables were significantly influenced by the trial type only on inclined floors $(P \leq 0.01)$ but not when walking on horizontal surfaces (NSL and SD). Finally, Forc Tdiff, $_{\text {, }}$ HeelVelx $_{\mathrm{HC}}$ and HeelAccx $\mathrm{HC}_{\mathrm{HC}}$ were not affected by trial type.

The ground reaction forces variables $\left(\mathrm{RCOF}_{\text {peak }}\right.$, Shear $r_{\text {peak }}$ and Norm $_{\text {peak }}$ ) were all significantly reduced by trial type (as shown by the positive differences from baseline values in Fig. 3). The anticipation trials produced peak RCOFs that were on average 16-33\% lower than those collected during the baseline trials, with more pronounced reductions as ramp angle was increased (Fig. 3a). The peak RCOFs during recovery trials were greater than the anticipation trials, but did not return to the levels of the baseline $(5-12 \%$ lower than baseline values). This trial type effect was found to

Table 2

Baseline normative values of gait parameters on vinyl tile and rough floors

\begin{tabular}{|c|c|c|c|c|c|c|}
\hline \multirow[t]{2}{*}{ Variable: mean (S.D.) } & \multicolumn{2}{|l|}{$0^{\circ}$} & \multicolumn{2}{|l|}{$5^{\circ}$} & \multicolumn{2}{|l|}{$10^{\circ}$} \\
\hline & Vinyl & Rough & Vinyl & Rough & Vinyl & Rough \\
\hline \multicolumn{7}{|l|}{ Kinetic parameters } \\
\hline $\mathrm{RCOF}_{\text {peak }}$ & $0.18(0.05)$ & $0.19(0.04)$ & $0.26(0.03)$ & $0.28(0.04)$ & $0.32(0.05)$ & $0.34(0.04)$ \\
\hline $\operatorname{Norm}_{\text {peak }}(\mathrm{N} / \mathrm{kg})$ & $10.92(1.42)$ & $10.73(0.84)$ & $12.15(1.41)$ & $11.90(1.06)$ & $13.33(1.52)$ & $13.06(1.64)$ \\
\hline Shear $_{\text {peak }}(\mathrm{N} / \mathrm{kg})$ & $1.77(0.61)$ & $1.80(0.52)$ & $2.94(0.56)$ & $3.03(0.64)$ & $4.06(0.81)$ & $4.23(0.85)$ \\
\hline $\mathrm{RCOF}_{\text {Tpeak }}(\%)$ & $16.5(2.4)$ & $16.4(2.8)$ & $18.1(3.6)$ & $17.0(2.8)$ & $19.2(4.6)$ & $18.5(4.1)$ \\
\hline Norm $_{\text {Tpeak }}(\%)$ & $24.5(5.2)$ & $23.9(4.7)$ & $21.4(4.3)$ & $22.6(4.3)$ & $18.6(5.4)$ & $18.9(5.6)$ \\
\hline Shear $_{\text {Tpeak }}(\%)$ & $19.0(3.1)$ & $18.6(3.0)$ & $19.5(2.6)$ & $19.6(3.5)$ & $19.0(4.6)$ & $18.7(4.6)$ \\
\hline Forc $_{\text {Tdiff }}(\%)$ & $5.5(3.8)$ & $5.3(4.2)$ & $2.0(3.6)$ & $3.0(3.1)$ & $-0.4(4.2)$ & $0.2(4.3)$ \\
\hline \multicolumn{7}{|l|}{ Kinematic parameters } \\
\hline NSL & $2.93(0.38)$ & $2.92(0.39)$ & $2.99(0.41)$ & $2.88(0.41)$ & $2.95(0.40)$ & $2.95(0.42)$ \\
\hline $\mathrm{SD}(\mathrm{ms})$ & $781.7(114.0)$ & $792.8(89.8)$ & $716.5(88.9)$ & $730.3(88.4)$ & $666.6(88.8)$ & $677.1(76.4)$ \\
\hline HeelVelx $_{\mathrm{HC}}(\mathrm{m} / \mathrm{s})$ & $0.19(0.39)$ & $0.15(0.31)$ & $0.25(0.42)$ & $0.10(0.23)$ & $0.13(0.32)$ & $0.01(0.23)$ \\
\hline HeelVelx $_{\text {rearward }}(\mathrm{m} / \mathrm{s})$ & $-0.11(0.07)$ & $-0.11(0.07)$ & $-0.09(0.08)$ & $-0.09(0.06)$ & $-0.09(0.09)$ & $-0.10(0.10)$ \\
\hline HeelVelx $_{\text {forward }}(\mathrm{m} / \mathrm{s})$ & $0.12(0.04)$ & $0.12(0.04)$ & $0.14(0.05)$ & $0.14(0.05)$ & $0.17(0.08)$ & $0.16(0.05)$ \\
\hline $\operatorname{HeelAccx}_{\mathrm{HC}}\left(\mathrm{m} / \mathrm{s}^{2}\right)$ & $-24.86(16.54)$ & $-26.69(13.72)$ & $-26.69(16.44)$ & $-22.79(11.0)$ & $-21.35(15.07)$ & $-18.10(10.62)$ \\
\hline $\operatorname{HeelAccx}_{\text {peak }}\left(\mathrm{m} / \mathrm{s}^{2}\right)$ & $4.01(2.34)$ & $4.33(2.25)$ & $3.45(2.18)$ & $3.45(1.62)$ & $3.04(1.98)$ & $2.85(1.70)$ \\
\hline FootAngVel $_{\mathrm{HC}}$ (degree per s) & $223.8(98.4)$ & $225.4(77.9)$ & $251.7(111.9)$ & $267.3(79.2)$ & $292.9(86.9)$ & $310.3(79.8)$ \\
\hline FootAngl $_{\mathrm{HC}}\left({ }^{\circ}\right)$ & $23.5(3.7)$ & $24.1(4.3)$ & $26.4(3.5)$ & $26.4(3.3)$ & $26.9(4.9)$ & $27.6(4.3)$ \\
\hline ShankAngl $_{\mathrm{HC}}\left({ }^{\circ}\right)$ & $105.3(3.4)$ & $105.6(3.2)$ & $107.2(2.4)$ & $106.8(2.1)$ & $107.8(2.2)$ & $107.7(2.5)$ \\
\hline
\end{tabular}



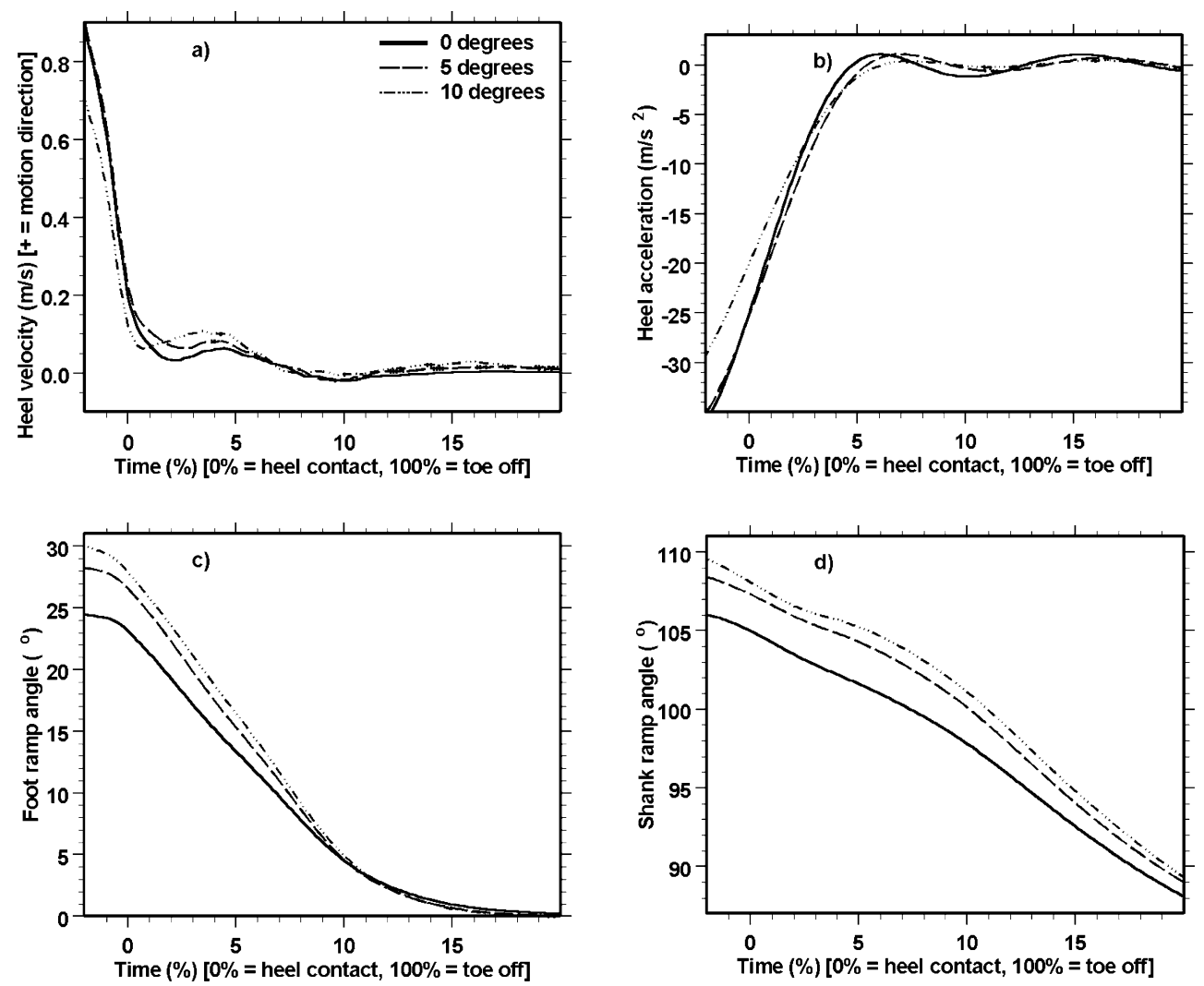

Fig. 2. Characteristic profile of the kinematic gait parameters $\left(0^{\circ}, 5^{\circ}, 10^{\circ}\right)$, averaged across all vinyl baseline trials, (a) heel velocity in the direction of motion; (b) heel acceleration in the direction of motion; (c) foot ramp angle and (d) shank ramp angle (time is truncated at $20 \%$ of stance for a more detailed view of heel contact dynamics).

be significant for all three ramp angles. Similarly, significant differences in the peak shear forces were also found, with the anticipation trials having significantly lower shears compared with the baseline $(17-40 \%$ difference) (Fig. 3b). The peak of the normal ground reaction forces was also reduced in the anticipation trials compared with the baselines $(2-13 \%)$, but to a lesser extent than shear forces when comparing relative changes to baseline conditions (Fig. 3c and Table 2).

When walking down inclined surfaces, the NSL (Fig. 4a) and SD (Fig. 4b) were both reduced during anticipation trials. However, there was no significant trial type effect on these variables during the level surface $\left(0^{\circ}\right)$ condition. On the inclined surfaces, small decreases from baseline values in the NSL ranged from 2 to $4 \%$ for the anticipation trials. Similar findings were recorded for the SD, with significant decreases of 3-6\% when comparing the anticipation with baseline trials on inclined surfaces. Despite these small changes, pairwise Tukey comparison tests indicated significant differences in these variables among all trial type conditions (on 5 and $10^{\circ}$ ) except for the comparison in the NSL between recovery and baseline trials on $5^{\circ}$.

The ANOVA results showed no significant differences in HeelVel $\mathrm{x}_{\mathrm{HC}}$ and HeelAccx $\mathrm{HC}_{\mathrm{HC}}$ among trial types. However, shortly after $\mathrm{HC}$, significant increases in the peak rearward heel velocity $\left(\right.$ HeelVel $\left._{\text {rearward }}\right)$ were recorded during anticipation trials (10 and 50-55\% increase from baseline values for level walking and inclined surfaces descent, respectively), and to a lesser degree during recovery trials $(10-20 \%$ increase), as depicted in the positive differences plotted in Fig. 5a. Before coming to a stop, the heel slid forward with reduced peak forward velocities during anticipation trials $\left(14-19 \%\right.$ reduction in HeelVelx $\mathrm{x}_{\text {forward }}$ from baseline characteristics for all ramp angles, Fig. 5b). No significant differences in HeelVel $\mathrm{x}_{\text {forward }}$ were detected between baseline and recovery conditions. Interestingly, during that short period of heel sliding after HC, anticipation of slippery surfaces led to significant increases in HeelAccx peak $(11,29$ and $40 \%$ for level walking, 5 and $10^{\circ}$, respectively) from magnitudes recorded during baseline trials (negative differences from baseline trials plotted in Fig. 5c). Differences in HeelAccx $x_{\text {peak }}$ between recovery and baseline trials were also significant, but lesser in amplitude (6-19\% relative increases during recovery trials, as depicted in Fig. 5c). These increases in $H_{e e l A c c x}$ peak are related to the greater HeelVel $x_{\text {rearward }}$ values reported earlier during anticipation trials, the duration of the short heel sliding period after $\mathrm{HC}$ is comparable for all conditions, however, greater rearward peak velocities were recorded for an- 
ticipation and recovery trials, thus leading to steeper $\Delta$ (velocity) $-\Delta$ (time) slopes or acceleration measures. In addition, decreases in FootAngVel ${ }_{\mathrm{HC}}$ were recorded during anticipation trials (positive differences from baseline conditions plotted in Fig. 5d) on all angles (decreases ranged from 0.5 to $23 \%$ with the smallest difference recorded on rough floors and the largest difference on vinyl tile surfaces). No significant differences in the FootAngVel $\mathrm{HC}_{\mathrm{C}}$ were detected between the recovery and baseline trials.

The anticipation and recovery trials were characterized by a significant decrease in the FootAngl $_{\mathrm{HC}}$ and ShankAngl $_{\mathrm{HC}}$ from baseline values for all ramp angle
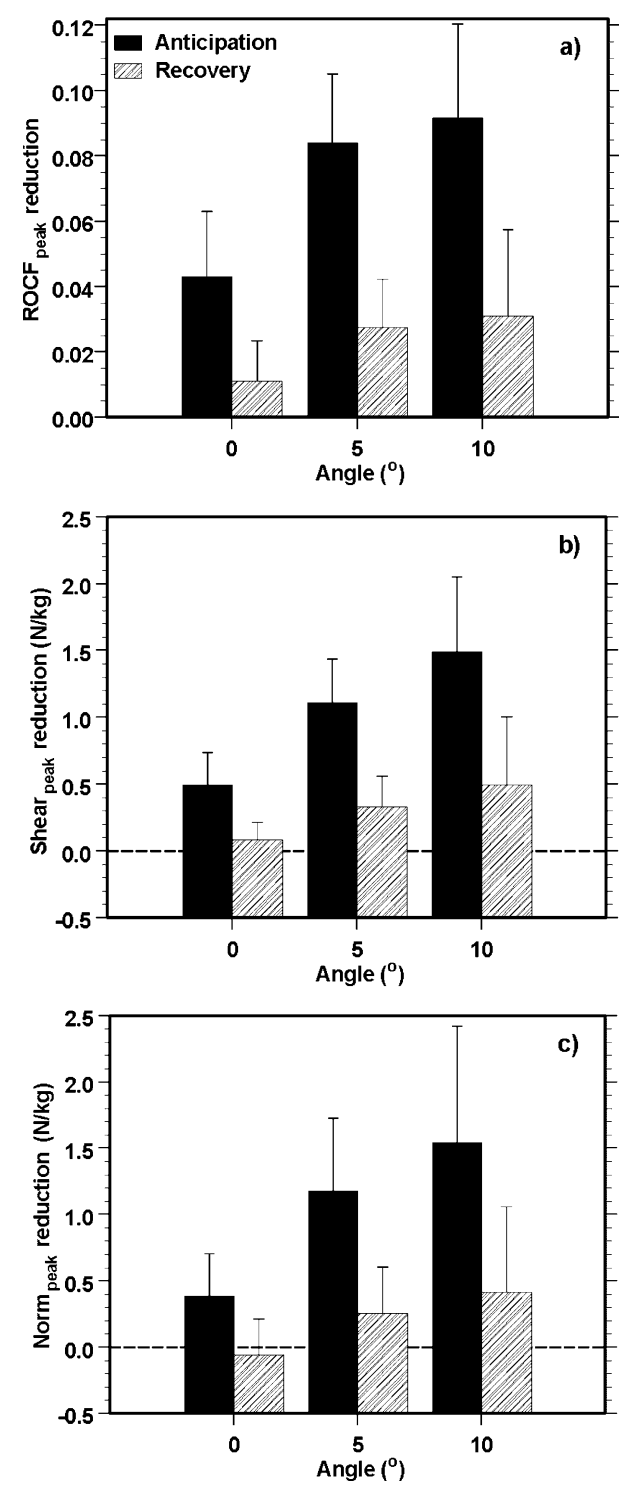

Fig. 3. Trial type effect on kinetic gait variables (data represent within-subject differences [baseline - anticipation/recovery] averaged across all subjects and flooring conditions), (a) peak required coefficient of friction; (b) peak anterior-posterior shear force and (c) peak normal force (dotted line indicates no-difference between baseline and anticipation/recovery trials). conditions (positive differences plotted in Fig. 5e and f). The foot orientation with respect to the floor was the most affected variable between the two (11 and 27\% decrease in FootAngl $\mathrm{HC}_{\mathrm{HC}}$ ). Less pronounced, but still statistically significant, reductions in the ShankAngl $\mathrm{HC}_{\mathrm{C}}$ were recorded $(1-3 \%)$. Postural adaptations were also evident in the knee and hip flexion increases during early stance of anticipation trials. In the recovery trials, these gait variables were closer to baseline characteristics with a decrease of $3-13 \%$ in the FootAngl $\mathrm{HC}_{\mathrm{HC}}$, and less than $2 \%$ in the ShankAngl $\mathrm{HC}_{\mathrm{HC}}$.

During the anticipation trials, the rate of loading on the supporting foot was significantly slower than for baseline trials. This is evident in the peak shear and peak normal force that were not recorded until 20-26 and $23-31 \%$ into stance for anticipation trials compared with $18-20$ and $19-25 \%$ for baseline trials, respectively (i.e., higher Shear ${ }_{\text {Tpeak }}$ and Norm Tpeak $_{\text {in the }}$ anticipation trials for all floor and ramp angle conditions). As a result, the peak RCOF also occurred later in the step for the anticipation trials $\left(\mathrm{RCOF}_{\mathrm{Tpeak}}\right)$, with similar delays as the shear forces. The differences in these timing variables $\left(\right.$ Shear $_{\text {Tpeak }}$, Norm Tpeak $_{\text {, }}$ $\mathrm{RCOF}_{\text {Tpeak }}$ ) were not always significantly different when performing pairwise Tukey comparison tests between recovery and baseline trials.

The trial type effect on the phase relationship between the normal and shear forces was investigated by examining the difference in timing between the peaks of the normal and shear forces among the trial type conditions. This variable ( Forc $\left._{\text {Tdiff }}\right)$ was not statistically different among trial types (all ramp angles), with less than $2 \%$ absolute differences with the baseline characteristics, indicating that the time relationship between the shear and normal ground reaction forces did not significantly change among trial types.

\subsection{Floor type effect}

Flooring condition had a small but statistically significant effect on the majority of the gait variables, while the interaction of the floor and trial type was not significant. The primary floor type influence on the kinetic variables was a higher $\mathrm{RCOF}_{\text {peak }}$ for the rough surface compared to the vinyl floor $(0.01-0.04$ overall difference) (Table 2 shows these differences for baseline values). This slight increase on rough floors was also true for the Shear ${ }_{\text {peak }}(0.1-0.4 \mathrm{~N} / \mathrm{kg}$ increase), whereas the Norm $_{\text {peak }}$ decreased on rough floors $(0.1-0.4 \mathrm{~N} / \mathrm{kg}$ decrease) for the majority of baseline and recovery conditions but increased for the anticipation trials (less than $0.3 \mathrm{~N} / \mathrm{kg}$ increase). Similarly, the kinematic variables were not greatly affected by the floor type, however, the changes were statistically significant. On average, recordings of the FootAngl $\mathrm{HC}_{\mathrm{HC}}$ were greater on rough floors compared to the vinyl surfaces for seven 

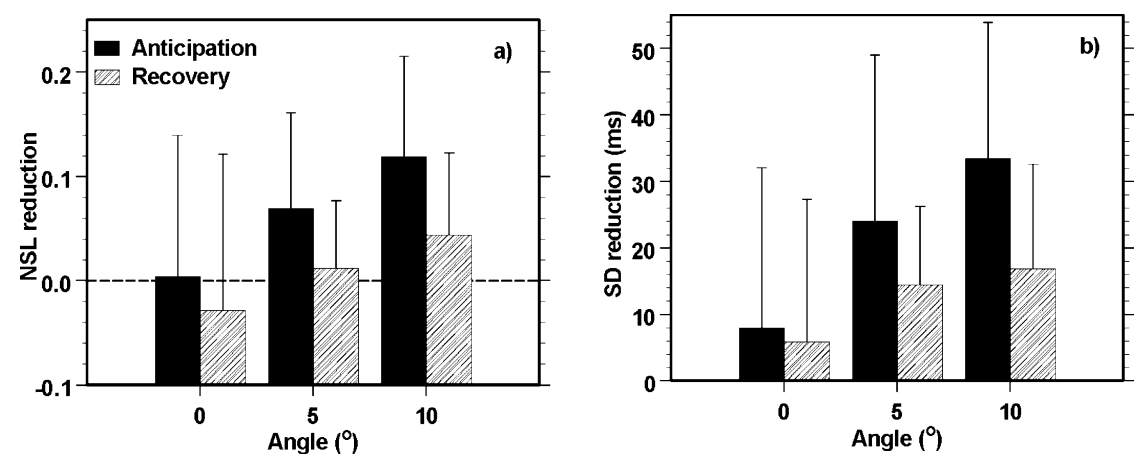

Fig. 4. Trial type effect on general gait variables (data represent within-subject differences [baseline - anticipation/recovery] averaged across all subjects and flooring conditions), (a) NSL; (b) SD (dotted line indicates no-difference between baseline and anticipation/recovery trials).

out of the nine (ramp angle, trial type) conditions. However, these variations in the foot orientation between the floor conditions were less than $2^{\circ}$. The findings related to the difference in the shank orientation between floors were less consistent across conditions and small in magnitude (less than $1^{\circ}$ ). HeelVelx $\mathrm{HC}_{\mathrm{HC}}$ was higher on vinyl surfaces compared with the rough floors (differences ranging from 0.04 to $0.23 \mathrm{~m} / \mathrm{s}$ for baseline and recovery trials and 0.18 to $0.34 \mathrm{~m} / \mathrm{s}$ for anticipation conditions), while the FootAngVel $\mathrm{HC}_{\mathrm{C}}$ was greater on rough floors (differences with vinyl surfaces ranged from 1 to $17^{\circ} / \mathrm{s}$ for baseline trials and from 26 to $50^{\circ} / \mathrm{s}$ for anticipation trials). In general, the floor effect on HeelAccx $_{\mathrm{HC}}$, HeelAccx peak $_{\text {, HeelVelx }}$ rearward and HeelVel $x_{\text {forward }}$ was not statistically significant.

\subsection{How was a reduction in the peak RCOF achieved?}

Given that the RCOF is an important variable in determining the potential for slips and falls, analyses were performed to investigate how the reduction of RCOF was achieved in the anticipation trials. In order to answer this question, a multivariate linear regression on the $\mathrm{RCOF}_{\text {peak }}$ with the independent variables being NSL, SD, FootAngVel $\mathrm{HC}_{\mathrm{HC}}$, FootAngl $_{\mathrm{HC}}$ and Norm $_{\text {Tpeak }}$ was repeated within each ramp angle condition. These variables were chosen for two reasons: (1) they significantly differed among trial types on one or more ramp angle conditions; and (2) they describe general gait variables, $\mathrm{HC}$ dynamics and time characteristics of gait.

$\mathrm{RCOF}_{\text {peak }}$ was significantly associated with slower loading rates $\left(\right.$ Norm $_{\text {Tpeak }}$ longer) and smaller foot-ramp angles at $\mathrm{HC}\left(\right.$ FootAngl $\left._{\mathrm{HC}}\right)$ on all ramp angles (Table $3)$. The peak RCOF increased with increasing normalized stride length (NSL), longer stance duration (SD) and slower $\mathrm{HC}$ angular foot velocity ( FootAngVel $_{\mathrm{HC}}$ ), with a stronger effect of these variables as ramp angle was increased. Interestingly, longer SDs were associated with higher peak normal forces ( $r$ between 0.6 and 0.7 ) and reduced peak forward heel accelerations ( $r$ between 0.5 and 0.6 ), while slower loading rates generated lower levels of shears ( $r$ between 0.5 and 0.6 ). Thus, subjects efficiently decreased their risk of slipping $\left(\mathrm{RCOF}_{\text {peak }}\right)$ by more slowly rotating the foot down onto the floor during $\mathrm{HC}$, adopting shorter strides (length and duration), reducing foot contact angles and slowing the transfer of body weight to the supporting foot.

\subsection{Joint moments}

\subsubsection{Baseline conditions}

During most of the stance period (after the loading phase), moments generated at the ankle, knee and hip joints were biased towards plantarflexion, extension and flexion, respectively. Lower extremity joint moments were affected by ramp angle. Early in stance ( $\sim 10 \%$ into stance), the peak dorsiflexion ankle moment during level walking was recorded at about 0.25 $\mathrm{N} \mathrm{m} / \mathrm{kg}$ for baseline trials, and increased by $25-30$ and $45-55 \%$ on 5 and $10^{\circ}$, respectively. After this time, the plantarflexion moment of the ankle increased to reach a local maximum of $1.0-1.3 \mathrm{~N} \mathrm{~m} / \mathrm{kg}$ (lower peak on inclined surfaces) before rapidly decreasing and returning to baseline levels (Fig. 6a). The knee was especially affected by ramp angle, with its maximum extension moment of $0.75 \mathrm{~N} \mathrm{~m} / \mathrm{kg}$ occurring later in the stance ( $21-24 \%$ into stance), increasing by $50-65 \%$ on $5^{\circ}$ and almost doubling when descending the $10^{\circ}$ ramp. After reaching this peak, the resulting knee moment decreased but stayed positive (extensor) during most of the stance with higher magnitudes on inclined surfaces (Fig. 6c). The hip moment was affected by ramp angle mostly in the $20-40 \%$ time interval of stance (Fig. 6e). Thus, it was evaluated at $30 \%$ into stance and found to increase on inclined surfaces $(0.6-0.8 \mathrm{~N} \mathrm{~m} / \mathrm{kg})$ from level walking characteristics $(0.42 \mathrm{~N} \mathrm{~m} / \mathrm{kg})$, with less pronounced differences (if any) between the 5 and $10^{\circ}$ ramp angles.

\subsubsection{Trial type effect}

ANOVA confirmed that the changes in the ground reaction forces, kinematic and temporal characteristics 
of gait induced by the anticipation trials led to statistically significant $(P<0.01)$ decreases in the peak moments developed at the ankle and knee and in the hip moment evaluated at $30 \%$ into stance. Knee and hip moments were most affected by the trial type condition (Fig. 6). Reductions in joint moments were more pronounced as ramp angle was increased (Fig. 7). More specifically, the mean baseline-anticipation difference in the peak dorsiflexion ankle moment decreased by about $27 \%$ during the anticipation conditions from baseline levels. The peak extension knee moment was associated with more significant differences between anticipation and baseline trials, leading to a relative reduction of $24 \%$ during level walking and about $35 \%$ when descending ramps. The anticipation conditions produced lower hip moment as well (evaluated of $30 \%$ ) with relative reductions of 32,49 and $59 \%$ on 0,5 and $10^{\circ}$, respectively. In addition to changes in its magnitude, the overall profile of the hip moment was somewhat modified during the anticipation trials (Fig. 5e and f). During the recovery trials, joint moments were closer but still lower than baseline characteristics. Pairwise Tukey comparison tests indicated statistically significant $(P<0.05)$ differences in all three joint moment variables among all trial types except between baseline and recovery trials on horizontal surfaces $(P<0.1)$.

The same ANOVA uncovered a significant floor effect on joint moment variables with, in general, slightly (but statistically significant) higher moments on rough floors compared to vinyl and smooth floors. Pairwise comparison tests indicated a number of negligible differences between the smooth and vinyl floors, in partic-
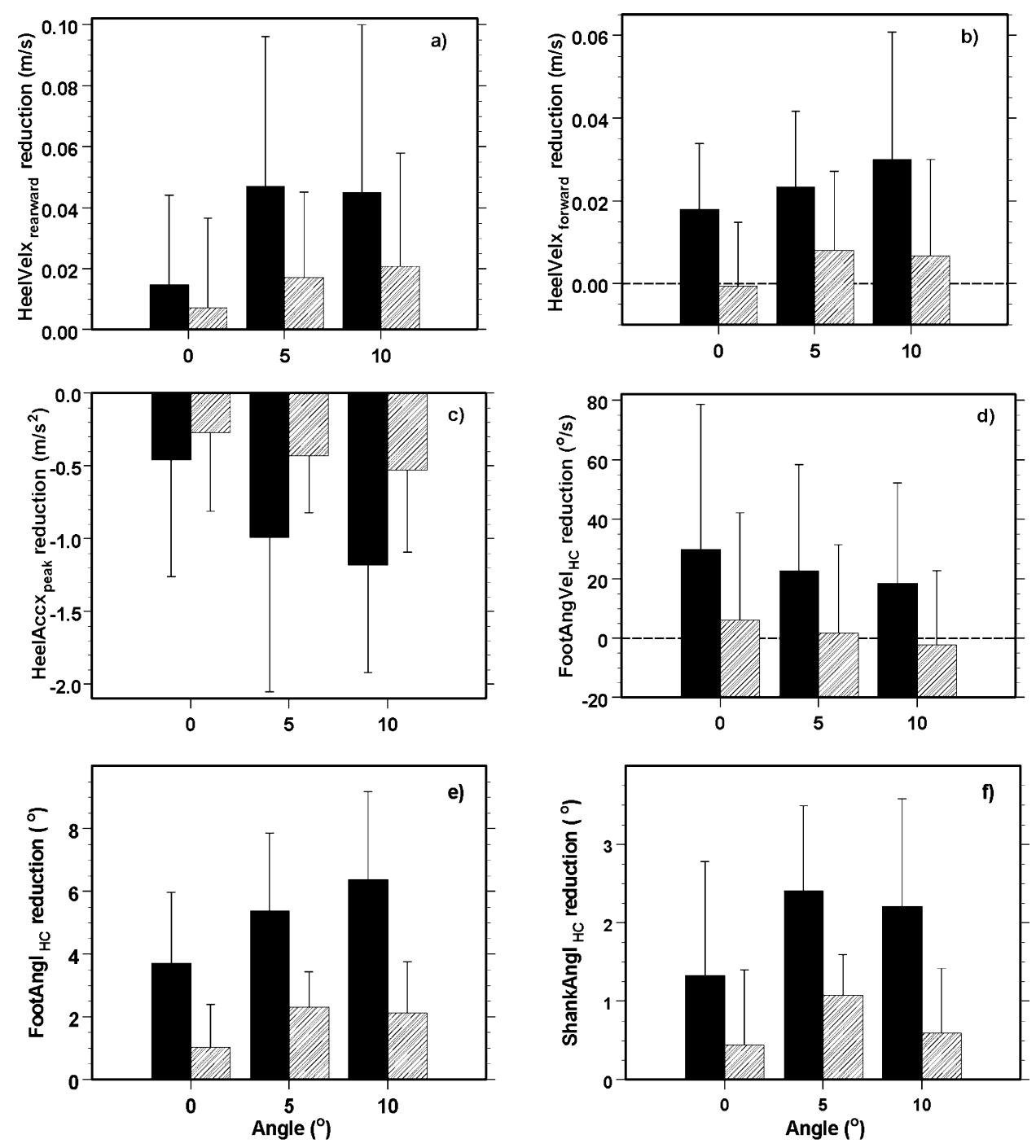

Fig. 5. Trial type effect on kinematic gait variables (data represent within-subject differences [baseline - anticipation/recovery] averaged across all subjects and flooring conditions), (a) peak rearward heel velocity; (b) peak forward heel velocity; (c) peak heel acceleration; (d) HC angular velocity of foot; (e) HC foot ramp angle and (f) HC shank ramp angle. (dotted line indicates no-difference between baseline and anticipation/recovery trials). Note, Heelvel rearward $_{\text {(negative) and HeelAcc }}$ peak (positive) increased with anticipation and recovery trials resulting in a positive and negative reduction in (a) and (c), respectively. 
Table 3

Gait characteristics associated the peak $\mathrm{RCOF}^{\mathrm{a}}$

\begin{tabular}{|c|c|c|c|c|c|}
\hline $\begin{array}{l}\text { Response }=\mathrm{RCOF}_{\text {peak }} \\
\text { Independent variable } \rightarrow \\
\text { Ramp angle }\left({ }^{\circ}\right) \downarrow\end{array}$ & NSL & SD & Footangvel $_{\mathrm{HC}}$ & Footangl $_{\mathrm{HC}}$ & Norm $_{\text {Tpeak }}$ \\
\hline 0 & NS & NS & NS (0.02) & $\mathrm{S}$ & $\mathrm{S}$ \\
\hline 5 & NS & NS & NS (0.06) & $\mathrm{S}$ & $\mathrm{S}$ \\
\hline 10 & S & $\mathrm{S}$ & S & $\mathrm{S}$ & S \\
\hline
\end{tabular}

${ }^{a} \mathrm{~S}$ indicates $P \leq 0.01$; NS indicates $P>0.01$.

ular for the hip moment. The interaction (trial type $\times$ floor type) was not significant for the majority of the tests.

\section{Discussion}

The main finding of this study was that human adaptations to 'potentially' slippery surfaces (anticipation trials) resulted in significant differences in gait biomechanics when compared with characteristics of baseline trials, during which subjects walked onto a known dry surface. The overall effect of these adaptations was a reduction in the peak RCOF, thus decreasing slip and fall potentials $[10-13,16,19,20]$. In addition, these adaptations led to significant reductions in joint moments. These two effects occurred despite instructions to the subjects to walk as normally as possible during both conditions.

Ultimately, the reaction forces at the shoe/floor interface are most important in determining the potential for slips and falls. The RCOF is believed to best reflect the aspects of the ground reaction forces contribution to slip and fall potential. Thus, the method by which the RCOF is reduced is important in determining how individuals control slip potential. How was a decrease in the peak RCOF achieved by the subjects in these experiments? Reductions in the relative magnitude of both the shear forces and, to a lesser extent, the normal forces, resulted in the overall effect of a decrease in the peak RCOF. Another possible way of affecting the peak RCOF is to change the time relationship between the shear and normal forces. However, interestingly, the phase relationship between the profiles of the shear and normal ground reaction forces did not vary among trial types. Thus the magnitude of the ground reaction force components appear to be adjusted and not the relative timing between the two. These magnitude changes in the kinetics of locomotion appear to occur due to temporal and kinematic gait adaptations including reductions in the SD and loading speed on the supporting foot, shorter NSL, reduced foot-ramp angle and slower angular foot velocity at HC. Thus, expectation of a potentially slippery surface led to more 'cautious' biomechanical gait patterns, which successfully resulted in a decrease of slip probability.

The overall statistical significance of anticipation effects is believed to be of practical relevance to the reduction in slip potential. This is evident in the extent of the frictional requirement reduction during anticipation conditions. For example, an approximate 0.09 reduction in $\mathrm{RCOF}_{\text {peak }}$ was recorded during the anticipation trials when descending the $10^{\circ}$ ramp, resulting in an absolute $\mathrm{RCOF}_{\text {peak }}$ value of about 0.23 , which is comparable to baseline magnitudes observed when descending the $5^{\circ}$ ramp. Thus, the consequences of biomechanical human reactions on slip potential during anticipation trials on the $10^{\circ} \mathrm{ramp}$ were equivalent to reducing the ramp angle by $5^{\circ}$. It is important to note, however, that the practical significance of anticipation was varied across gait variables, with subtle changes in general gait variables (e.g. NSL and SD) and more pronounced effects on postural responses (foot orientation), which are believed to be related to important consequences such as decreases in shear forces and thus reduction in slip potential.

The results of this study concerning the floor effect are consistent with the hypothesis that subjects were aware of the greater possibility of slipping on vinyl floors compared with rough floors, and, therefore, adapted their gait to achieve once again a reduction in the peak RCOF on the 'more dangerous' surfaces. However, the possibility of a contaminated surface affected gait patterns more than floor type. In general, floor effect on gait variables was significant but small, while the interaction of floor type and trial type was not statistically significant.

The baseline results reported here are comparable to findings of earlier studies. In particular, peak RCOFs were in close agreement with values reported by Redfern and Dipasquale [11] and Hanson et al. [12] on both horizontal and inclined surfaces, and by Perkins [10] and Strandberg and Lanshammar [13] on level floors. Similarly, the timing and magnitude of peak ground reaction forces were in accordance with the same above-mentioned studies and results of baseline gait experiments conducted by Winter [21] on level surfaces. In general, the overall shape of the calculated joint 
moments were comparable and within the range of values reported by Redfern and Dipasquale [11] and Winter [21], although some differences were noted in the magnitudes. Potential reasons for these differences may include minor differences in marker placement and other experimental factors such as specific instructions given to subjects. Despite these differences, the relative differences between the three trial types remain.

Baseline kinematic data were also comparable to earlier published findings. For example, joint angles during walking on level and inclined surfaces agreed with results of Redfern and Dipasquale (on level and inclined surfaces) [11] and Winter (on level surfaces) [21]. The wide variation in $\mathrm{HC}$ heel velocity patterns observed among subjects was also reported by Strandberg and Lanshammar [13] with velocity magnitudes ranging from 0.14 to $0.68 \mathrm{~m} / \mathrm{s}$ and associated with S.D. as large as $0.52 \mathrm{~m} / \mathrm{s}$, a finding that certainly agrees with the results of this study. A final example related to the $\mathrm{HC}$ foot orientation with respect to the floor published by Strandberg and Lanshammar [13] on horizontal surfaces $\left(22.0 \pm 5.3^{\circ}\right)$, which is also in agreement with values computed here. When comparing kinematic characteristics of steps prior to and onto a knowingly slippery area, Andres et al. [18] have reported similar gait adaptations than those reported here, including reduced foot angle and angular foot velocity at $\mathrm{HC}$.

The findings of this study provide a better understanding of important aspects related to slips and falls biomechanics. First, humans have the ability to reduce slip probability on potentially contaminated floors. Second, in order to achieve this reduction, it appears that postural and temporal gait adaptations were adopted to decrease ground reaction forces, resulting in smaller RCOFs. Third, these adaptations did not lead to phase changes between the normal and shear foot floor forces.
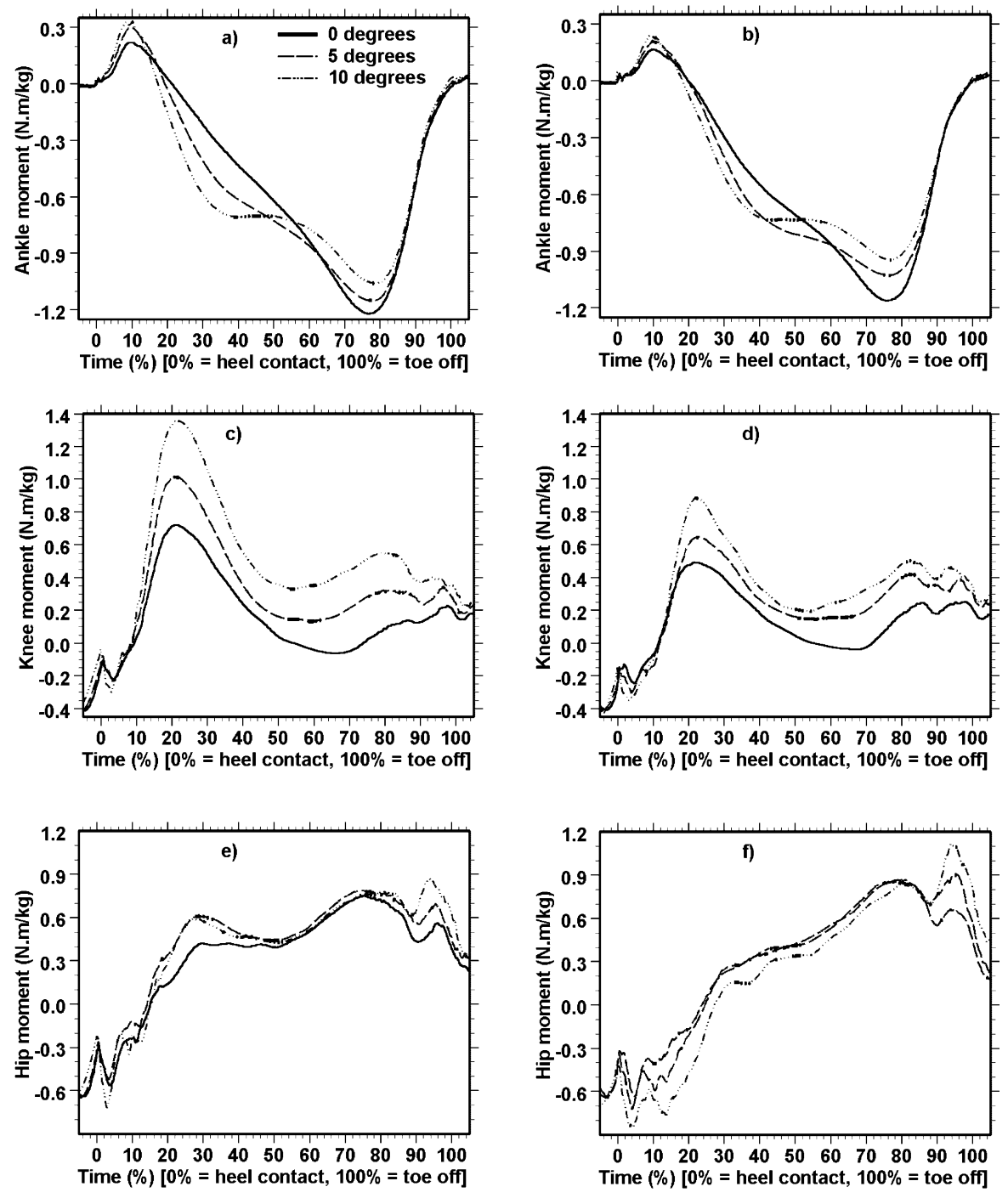

Fig. 6. Characteristic profile of lower extremity joint moments (normalized to body weight) during gait on the vinyl tile floor $\left(0,5,10^{\circ}\right)$, averaged across all baseline (left) and anticipation (right) trials, (a) ankle $[+=$ dorsiflexion], (b) knee $[+=$ extension $]$, and (c) hip $[+=$ flexion $]$. 

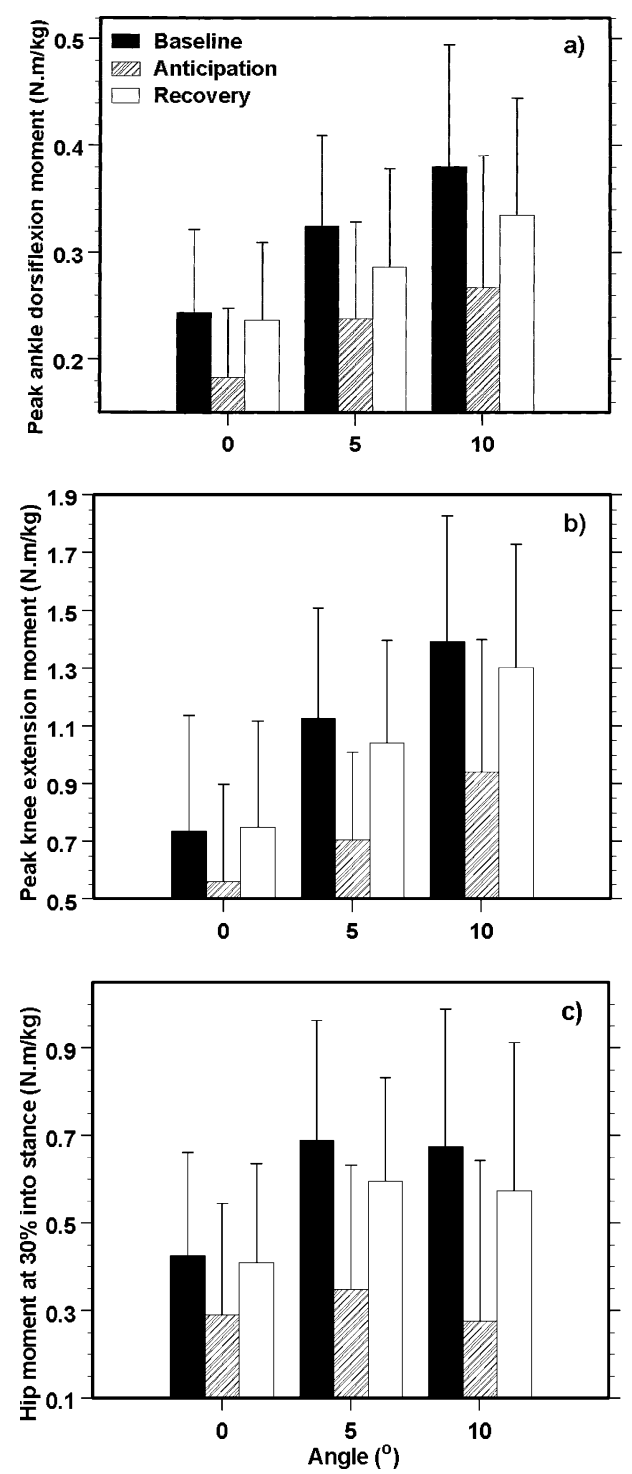

Fig. 7. Trial type effect on joint moments parameters (normalized to body weight) $\left(0,5,10^{\circ}\right)$, averaged across all subjects and floors, (a) peak ankle dorsiflexion moment; (b) peak extension knee moment and (c) hip moment evaluated at 30\% into stance.

Finally, as a result of changes in ground reaction forces and postural adjustments during walking, anticipation of slippery surfaces affected lower extremity joint moments, a reflection of overall muscle reactions. In addition, the findings related to the moments at the lower extremity joints suggested that the knee and hip appear to be used more than the ankle to control slip potential.

The results of this study have at least two possible applied implications in future slip and fall experiments using human subjects. First, recruiting subjects for such experiments involves making them aware of the possibility of slipping; as a result of this expectation of potential floor contamination, subjects will walk 'more carefully', thus modifying baseline gait characteristics to decrease slips/falls potentials; the extent of change in gait biomechanics will depend on the a-priori descrip- tion of the study and specific instructions to the subject, thus emphasizing the need of being consistent throughout the trials. Second, reproducing the unexpected conditions often encountered in real life slips and falls incidents has proven to be very difficult, therefore, the conclusions reported here underline the importance of being conservative when (1) applying research findings from human subjects slip and fall experiments to design criteria of 'safe' floor-foot interfaces and (2) providing gait variables as input to slip resistance testers designed to reproduce foot motion. Finally, a potential safety implication of the findings of this paper is that proper safety warnings of slippery environments would result in similar gait changes as those associated with anticipation effects, thus decreasing slip potential.

The main finding of this study was that human adaptations to 'potentially' slippery surfaces (anticipation trials) resulted in significant differences in gait biomechanics when compared with characteristics of baseline trials, during which subjects walked onto a known dry surface. The overall effect of these adaptations was a reduction in the $\mathrm{RCOF}_{\text {peak }}$, thus humans have the ability to reduce slip potential on possibly contaminated floors. Findings related to the moment generated at the lower extremity joints suggested that the knee and hip appear to be used more than the ankle to control slip potential.

\section{Acknowledgements}

Supported by NIOSH (5 R03 OH03621).

\section{References}

[1] McCraig LF, Stussman BJ. National hospital ambulatory medical care survey: 1996 emergency department summary. Vital and Health Statistics of the Centers for Disease Control and Prevention-National Center for Health Statistics, no. 293; 1997. p. $1-20$.

[2] Rice DP, MacKenzie EJ. Cost of injury in the US: a report to Congress. Institute for Health and Aging, University of California and Johns Hopkins University Press; 1989.

[3] Englander F, Hodson TJ, Terregrossa RA. Economic dimensions of slip and fall injuries. J Forensic Sci 1996;41(5):733-46.

[4] United States Department of Labor. Bureau of Labor Statistics, 1998.

[5] Fingerhut LA, Cox CS, Warner M. International comparative analysis of injury mortality; findings from the ICE on injury statistics. Vital and Health Statistics of the Centers for Disease Control and Prevention-National Center for Health Statistics 1998, no. 303 , p. $1-19$.

[6] Lloyd DG, Stevenson MG. Investigation of floor surface profile characteristics that will reduce the incidence of slips and falls. Mech Eng Trans Inst Eng 1992;ME17(2):99-105.

[7] Bell JL, Gardner LI, Landsittel DP. Slip and fall-related injuries in relation to environmental cold and work location in aboveground coal mining operations. Am J Ind Med 2000;38:40-8. 
[8] Bentley TA, Haslam RA. Slip, trip and fall accidents occurring during the delivery of mail. Ergonomics 1998;41(12):1859-72.

[9] Björnstig U, Björnstig J, Dahlgren A. Slipping on ice and snow-elderly women and young men are typical victims. Accid Anal Prev 1997;29(2):211-5.

[10] American Society for Testing and Materials, Perkins PJ. Measurement of slip between the shoe and ground during walking. In: Walkway Surfaces Measurements of Slip Resistance, vol. 649. ASTM Special Technical Publication, 1978:71-87.

[11] Redfern MS, DiPasquale J. Biomechanics of descending ramps. Gait Posture 1997;6(2):119-25.

[12] Hanson JP, Redfern MS, Mazumdar M. Predicting slips and falls considering required and available friction. Ergonomics 1999;42(12):1619-33.

[13] Strandberg L, Lanshammar H. The dynamics of slipping accidents. J Occup Accid 1981;3:153-62.

[14] Tang PF, Wollacott MH. Inefficient postural responses to unexpected slips during walking in older adults. J Gerontol Med Sci 1998;53(A):M471-80.

[15] Grönqvist R, Roine J, Järvinen E. An apparatus and a method for determining the slip resistance of shoes and floors by simulation of human foot motions. Ergonomics 1989;32(8):979-95.
[16] Redfern MS, Bidanda B. Slip resistance of the shoe-floor interface under biomechanically-relevant conditions. Ergonomics 1994;37(3):511-24.

[17] Wilson MP. Development of SATRA slip test and tread pattern design guidelines. American society for testing and materials. In: Slips, Stumbles, and Falls: Pedestrian Footwear and Surfaces, vol. 1103. ASTM Special Technical Publication, 1990:113-23.

[18] Andres RO, Oconnor D, Eng T. Practical synthesis of biomechanical results to prevent slips and falls in the workplace. In: Advances in Industrial Ergonomics and Safety IV, 1992:1001-6.

[19] Buczek FL, Cavanagh PR, Kulakowski BT, Pradhan P. Slip resistance needs of the mobility disabled during level and grade walking. American society for testing and materials. In: Slips, Stumbles, and Falls: Pedestrian Footwear and Surfaces, vol. 1103. ASTM Special Technical Publication, 1990:39-54.

[20] Buczek FL, Banks SA. High-resolution force plate analysis of utilized slip resistance in human walking. $\mathbf{J}$ Testing Eval 1996;24(6):353-8.

[21] Winter DABC. (Anatomy, biomechanics and control) of balance during standing and walking. In: Waterloo Biomechanics. Graphic Services, University of Waterloo, 1995. 